\title{
When developing a neuroleptic cardiomyopathy?: morphometric study of myocardium
}

\begin{abstract}
By a morphometric method of research the pathologic changes of the myocardium in the process of antipsychotic therapy and in neuroleptic cardiomyopathy was determined. The aim of this work was to study an issue about the timing of this disease. The analysis of the obtained results shows that the development of neuroleptic cardiomyopathy begins gradually after ten years of psychotropic treatment. Clinical manifestations of the disease occur after 20years of taking antipsychotics due to the compensatory-adaptive processes occurring during this period in the myocardium.
\end{abstract}

Keywords: antipsychotics, cardiotoxicity, duration of antipsychotic therapy, neuroleptic cardiomyopathy, pathomorphology of myocardium, morphometry
Volume 12 Issue 4 - 2019

\author{
Vladimir PVolkov \\ Tver Center of Judicial Examinations, Russia
}

Correspondence: Vladimir PVolkov, Tver Center of Judicial

Examinations, A. Zavidov st., 24,

of. 6, I70008,Tver, Russia, Tel. +7-9|5-70I-58-68,

Email patowolf@yandex.ru

Received: June II, 2019 | Published: August 08, 2019

\section{Introduction}

Neuroleptic cardiomyopathy (NCMP) develops due to the side cardiotoxic action of antipsychotic (neuroleptic) medications and is one of the most dangerous complications of antipsychotic therapy (APT).$^{1-4}$ Demonstration NCMP occurs usually after prolonged treatment with antipsychotics. ${ }^{5,6,7}$ For example, according to the systematic literature review conducted by Alawami M et al., ${ }^{8}$ after the start of receiving clozapine, perhaps the most cardiotoxic antipsychotic drug, symptoms of the disease appear after an average of 14.4months. B. Makhoul and colleagues (2008). ${ }^{5}$ describe a patient even with seven years of experience using this antipsychotic. Overall, however, the timing of the disease is still not exactly specified. In this regard, it is of considerable scientific and practical interest to study this issue, using the morphometric method of studying the myocardium, which meet the modern requirements of evidence-based medicine. ${ }^{9}$, ${ }^{10}$ and allow objectifying the results and conclusions, since the final values of the parameters under study are quantitative and easy enough to be statistically analyzed. ${ }^{11,12}$

\section{Material and methods}

I $t$ is known that a quantitative morphologic characteristic of changes of each organ in the case of its any pathology must start from a definite "reference point" which is defined by the concept of a "norm". ${ }^{13}$ Therefore at the research beginning the results of autopsy protocols of 100 persons (50 men and 50 women) in the age from 18 to 82 years were analyzed who not have a concomitant cardiac pathology and died of non-cardiac causes (group I). The myocardial parameters, which were received in this group, were taken as relative norm (RN). To characterize cardiac changes in NCMP at the microscopic (tissue and cellular) level, morphometry of myocardium was performed in 58 deceased patients with schizophrenia (38 men and 20 women; age from 16 to 77 years), who suffered during the life of NCMP, verified at autopsy (group II). Finally autopsy protocols of 70 patients with schizophrenia (41 men and 29 women) who died at the age from 22 to 77 years were analyzed. The final diagnosis of each deceased was verified at the autopsy. The criteria of an exception were the expressed signs of a metabolic syndrome (the increased body weight, arterial hypertension, a diabetes mellitus), a chronic pulmonary pathology with hypertension in a small circle of blood circulation, a cachexia. During their lives the patients received various antipsychotic in quantities corresponding to the therapeutic standard; these medicines are not rarely received in combination with each other. The duration of APT ranged from six months to 30 years or more. Depending on the duration of the APT material is divided into four groups (III-VI): III- up to ten years (20 dead); IV-from 11 to 20years (25); V-from 21 to 30years (19); VI-over 30years (6). Microscopy and micromorphometry of the myocardium (tissue and cellular levels) are carried out according to the proposed for this purpose own algorithm. ${ }^{14,15}$

Myocardium slices from various departments of the left ventricle were filled in paraffin, cuts were painted by hematoxylin and eoziny. Respective objects were studied in 10 different fields of microscope, with necessary magnifications with the help of an ocular micrometer, the point count method was also used. ${ }^{11,12,16}$ Such parameters as zone of pericapillary diffusion (ZPD), Kernogan index (KI), stromal-parenchymatous ratio (SPR), rate of interstitial edema (RIE) were calculated. Karyometry and cytometry of cardiomyocytes (CMC) were performed, the specific volumes of hypertrophied CMC (SVHC), of atrophied ones (SVAC), and-by the method of polarization microscopy - the specific volume of dystrophic ones (SVDC) were determined. The method of polarization microscopy was used to detect dystrophic-degenerative changes in CMC. It is believed that the combination of this type of study with conventional histological and histochemical techniques allows to obtain and evaluate much more complete information about the state of CMC and their myofibrillar apparatus, which is a very sensitive indicator of myocardial damage..$^{17,18}$ This makes the polarization microscopy method most suitable for detecting early stages of CMC damage. ${ }^{17}$ The above-named parameters describe a condition of three structural components of myocardium: of microvasculature (ZPD and $\mathrm{KI}$ ), intercellular matrix (SPR and RIE), and parenchyma (SVHC, SVAC and SVDC). The obtained quantitative results were processed statistically (computer program "Statistica 6.0") with the level of significance of differences of $95 \%$ and more $(\mathrm{p} \leq 0.05)$.

\section{Results}

The data obtained during the study are presented in Table 1 . 
Table I Microscopic changes of the myocardium at NCMP and in the process of APT

\begin{tabular}{|c|c|c|c|c|c|c|c|}
\hline \multirow{2}{*}{$\begin{array}{l}\text { Indicators } \\
\text { Groups }\end{array}$} & \multicolumn{2}{|c|}{ Microvasculature } & \multicolumn{2}{|c|}{ Intercellular matrix } & \multicolumn{2}{|c|}{ Cardiomyocytes } & \multirow[b]{2}{*}{ SVDC } \\
\hline & ZPD & $\mathbf{K} \mathbf{I}$ & SPR & RIE & SVHC & SVAC & \\
\hline I & $111.3 \pm 17.9 * *$ & $1.22 \pm 0.10 * *$ & $8.1 \pm 5.0 * *$ & $7.1 \pm 4.6 * *$ & $10.2 \pm 5.0 * *$ & $4.8 \pm 3.6 * *$ & $2.2 \pm 2.6 * *$ \\
\hline II & $246.5 \pm 70.8 *$ & $1.62 \pm 0.18^{*}$ & $58.8 \pm 5.3^{*}$ & $60.7 \pm 5.1 *$ & $25.8 \pm 4.9 *$ & $35.2 \pm 5.3^{*}$ & $25.3 \pm 4.7^{*}$ \\
\hline III & $128.5 \pm 24.0 * *$ & $1.32 \pm 0.11 * *$ & $10.3 \pm 5.8^{* *}$ & $9.8 \pm 5.6 * *$ & $16.9 \pm 7.2^{* *}$ & $8.4 \pm 5.3^{* *}$ & $5.7 \pm 4.4^{* *}$ \\
\hline IV & $\mid 79.7 \pm 46.7^{*}$ & $1.5 \mid \pm 0.19 *$ & $41.8 \pm 8.6^{*}$ & $37.7 \pm 8.2^{* * *}$ & $37.0 \pm 8.5 * * *$ & $23.9 \pm 7.4 * * *$ & $13.6 \pm 6.0 * * *$ \\
\hline $\mathrm{V}$ & $263.2 \pm 73.1^{*}$ & $1.64 \pm 0.15^{*}$ & $63.4 \pm 9.3^{*}$ & $72.3 \pm 8.9 * * *$ & $19.6 \pm 7.9 *$ & $39.7 \pm 9.8^{*}$ & $28.5 \pm 9.0^{*}$ \\
\hline VI & $316.4 \pm 83.7^{*}$ & $\mid .72 \pm 0.2 \mathrm{I} *$ & $80.0 \pm 10.1 * * *$ & $83.4 \pm 9.4 * * *$ & $17.0 \pm 9.5$ & $45.1 \pm 12.6^{*}$ & $35.2 \pm 12.1 *$ \\
\hline
\end{tabular}

*-statistically significant difference with group I, **-statistically significant difference with group II.

\section{Discussion}

The analysis of the obtained results shows that in case of NCMP in a myocardium (at the tissue and cellular levels of cardiac organization) statistically significant differences with $\mathrm{RN}$ in all studied morphometric parameters are found. The noted pathological shifts in the morphometric parameters of the myocardium, reflecting the deep tissue changes in the heart muscle dystrophic-degenerative, atrophic, sclerotic, as well as compensatory-adaptive nature, ${ }^{15,19-21}$ unfolding in the process of implementing the cardiotoxic effect of antipsychotic drugs, are briefly summarized as follows. First of all, significant changes occur in the quantitative characteristics of the relationship of the parenchyma of the myocardium and the metabolic level of microvasculature, which clearly show a statistically significant and very sharply increasing in comparison with the $\mathrm{RN}$ the magnitude of the ZPD and KI (growth is respectively $121.5 \%$ and $32.8 \%$ ). These findings indicate deep microcirculation disorders in the myocardium due to cardiotoxicity of antipsychotics. With these hemodynamic changes are closely related to the progression of the interstitial edema of the myocardium and increase the severity of myofibrosis (growth of indicators RIE and SPR reaches respectively $754,9 \%$ and $625,9 \%$ ). Both of these pathological processes, unfolding in the intercellular matrix of the heart muscle, lead to the separation of the nutritive blood capillaries and $\mathrm{CMC}$, which seriously upsets the trophic of the latter and leads to their severe damage. ${ }^{17,19-22}$ Discovered that the number of hypertrophic cardiomyocytes increases dramatically, but to an even greater extent increases the number of atrophied cardiomyocytes (growth of indicators SVHC and SVAC is $152,9 \%$ and $633,3 \%$ respectively). At the same time, the process of dystrophic-degenerative changes in CMC is rapidly progressing-the value of SVDC increases by more than an order of magnitude. As follows from the analysis of other data in Table 1, with the increase in the duration of APT, the ratio of tissue components of the heart muscle varies significantlystatistically significant differences with the relative norm can be traced after ten years of taking of antipsychotics (IV-VI group).

The direct consequence of the cardiotoxic action of antipsychotics is clearly expressed microcirculation disorders in the myocardium, which is indicated by significantly increasing values of ZPD and KI compared to RN. Discirculatory disorders cause changes in the microvasculature and intercellular matrix of the myocardium in the form of increase of interstitial edema and the development of myofibroses that documents the increase in RIE and SPR. As mentioned earlier, these pathological processes lead to the separation of the nutritive blood capillaries and $\mathrm{CMC}$, seriously upsetting the cell trophic and leading to severe damage of $\mathrm{CMC},{ }^{17,19-22}$ Under the influence of antipsychotics, the number of hypertrophied CMC (level of SVHC) is subject to directional fluctuations, reaching a maximum in group IV, and then significantly decreasing. Such dynamics is a reflection of the compensatory-adaptive processes occurring in the myocardium in the initial and medium-term stages of APT and gradually fading in the future, indicating the depletion of the adaptive capacity of the heart muscle. On the contrary, increasing the time of exposure up the number of atrophied and dystrophic cardiomyocytes has been steadily and significantly growing that documents the changes of such indicators as SVAC and SVDC. These processes are a standard sign of progredient myocardial dysfunction. ${ }^{17,19-23}$ Having received data on morphometric changes of the myocardium in NCMP and in the process of APT, we came up to the main goal of the studyto determine the approximate timing of taking antipsychotics, in which there is a threat of the development of such a severe iatrogenic pathology as NCMP. To do this, we consistently compare these changes in the NCMP (group II) with those in different periods of APT (group III-VI).

The analysis carried out in this direction shows that the differences between groups II and III are statistically significant for all indicators. The marked differences between groups II and IV relate to four of the seven indicators, which characterize the state of the CMC. However, in group II there are no differences in the vast majority of parameters compared to those in subsequent groups of observations (V-VI). In other words, the morphometric changes of the heart muscle observed in the morphogenesis of NCMP and after twenty years of APT are almost identical. However, certain features peculiar to NCMP, in particular, dyscirculatory disorders and the development of myofibrosis, myocardium acquires after ten years of taking antipsychotic drugs. In the next decade, thanks to adaptive processes, the pumping function of the heart is not significantly disturbed-the NCMP is in a latent preclinical stage. ${ }^{24}$ Only after a twenty-year period of APT myocardial changes go so far as to develop myocardial dysfunction, and the disease goes first to the expanded stage, and then to the terminal, accompanied by the progression of congestive heart failure. $^{24}$

\section{Conclusion}

Morphometric approach to the study of myocardium in different periods of APT showed that the development of severe iatrogenic complications-NCMP due to the side cardiotoxic effects of antapsychotic drugs begins gradually after ten years of psychotropic treatment. Clinical manifestations of the disease occur much later (after twenty years of taking antipsychotics. 


\section{Acknowledgments}

None.

\section{Conflicts of interest}

Authors declare that there are no conflicts of interest.

\section{References}

1. Volkov VP. Cardiotoxicity of phenothiazine neuroleptics (review of literature). Psichiat psychopharmacother. 2010;12(2):41-45.

2. Volkov VP. Phenothiazine dilated cardiomyopathy: some aspects of clinic and morphology. Clin Med. 2009; 87(8):13-16.

3. Buckley NA, Sanders P. Cardiovascular adverse effects of antipsychotic drugs. Drug Saf. 2000;23(3):215-228.

4. Coulter DM, Bate A, Meyboom RHB, et al. Antipsychotic drugs and heart muscle disorder in international pharmacovigilance: data mining study. Br Med J. 2001; 322(7296):1207-1209.

5. Makhoul M, Hochberg I, Rispler S et al. Dilated cardiomyopathy: an unusual complication of clozapine therapy. Nat. Clin. Pract. Cardiovasc. Med. 2008;5(9):566-570.

6. Tanner MA, Culling W. Clozapine associated dilated cardiomyopathy. Postgrad. Med. J. 2003;79(933): 412-413.

7. De Berardis D, Serroni N, Campanella $D$ et al. Update on the adverse effects of clozapine: focus on myocarditis. Curr. Drug Saf. 2012;7(1):5562.

8. Alawami M, Wasywich C, Cicovic A et al. A systematic review of clozapine induced cardiomyopathy. Int. J. Cardiol. 2014;176(2):315-320.

9. Grinkhalkh T. The basics of evidence based medicine. lane with English Moscow: GEOTAR-MED publ; 2004.

10. Klyushin DA, Petunin YuI. Evidential medicine. Application of statistical methods. Moscow: Dialectics Publ; 2008.
11. Avtandilov GG. Fundamentals of quantitative pathological anatomy. Moscow: Medicine Publ; 2002.

12. Avtandilov GG. Medical morphometry: management. Moscow: Medicine Publ; 1990.

13. Petlenko VP, Tsaregorodtsev GI. Philosophy of medicine. Kiev: Zdorovja Publ; 1979

14. Volkov V. Cardiotoxicity of neuroleptics: etudes of studies of the problem. Beau Bassin, LAP Lambert Acad. Publ. 2018.

15. Volkov VP. Quantitative pathomorphology of specific dilated cardiomyopathies. Tver: Triada Publ; 2016.

16. Gutsol AA, Kondratyev BYu. Practical morphometry of organs and tissues. Tomsk: Tomsk Univer Publ; 1988.

17. Nepomnyashchih LM. Morphogenesis of the most important general pathological processes in the heart. Novosibirsk, Nauka Publ. 1991.

18. Cellarius $Y u$, Semenova LA. Histopathology of focal metabolic myocardial damage. Novosibirsk: Science Publ; 1972.

19. Volkov VP. Morphological characteristic of a neuroleptic cardiomyopathy Aktualnye voprosy patologicheskoj anatomii i sudebnoj mediciny. Novosibirsk: SibAK Publ; 2013. p. 50-85.

20. Volkov V. Morphological characteristics of neuroleptic cardiomyopathy. Vrach. 2013;5:86-88.

21. Volkov VP. Morphometric aspects of a morphogenesis of an antipsychotic cardiomyopathy. Ros J Cardiol. 2012;3(2):68-73.

22. Shumakov VI, Hubutiya MSh, Ilyinsky IM. Dilated cardiomyopathy. Tver: Triada Publ; 2003.

23. Amosova EN. Cardiomyopathies. Kiev: Kniga plyus Publ; 1999.

24. Volkov VP. Clinical characteristic of an antipsychotic cardiomyopathy. In: Volkov VP, editor. Aktual'nye problemy terapevticheskoj kliniki: kollektivnaya nauch. monografiya. Novosibirsk: SibAK Publ; 2013. p. 94-116. 